\title{
RIGIDITY OF GENERALIZED UNISERIAL AND FROBENIUS ALGEBRAS
}

\author{
S. S. PAGE
}

Introduction. In [2], [4] Gerstenhaber, Nijenhuis and Richardson introduced the concept of deformations of an algebra $A$ over a field. In this note we consider the question of whether there are any reasonable classes of algebras which admit nontrivial deformations but for which all the deformations remain in the class. The main theorem states that if $A$ is a generalized uniserial basic algebra, then every deformation of $A$ is generalized uniserial. The theorem actually gives a complete description of all deformations of a generalized uniserial basic algebra.

The second theorem states that the class of Frobenius algebras is a class closed under deformations.

We begin by setting up the notation. Throughout $A$ will denote an associative algebra over a field $k$ which admits a Wedderburn decomposition, $A=S+N$ where $S$ is $K$ separable and $N$ is the Jacobson radical. $A$ will be called a basic algebra if the simple components of $A$ are one dimensional over $k$ and in this case we will write $S=\sum_{i=1}^{n} k e_{i}$ where $e_{i}$ is the identity of the $i$ th component of $S$.

A generalized uniserial algebra $A$ is an algebra such that for any primitive idempotent $e$ the left (resp. right) modules $A e(e A)$ have a unique decomposition series.

Following [2], $A_{0}=A \otimes_{k} k((t))$ where $k((t))$ is the field of quotients of the power series ring over $k$ in one indeterminant $t$. By a deformation of $A$ we will mean an associative multiplication induced by a bilinear function $f_{t}: A \otimes_{k} A \rightarrow A_{0}$ of the form

$$
f_{t}(a, b)=a b+t F_{1}(a, b)+t^{2} F_{2}(a, b) \cdots .
$$

Call such functions $f_{t}$ multiplicative functions. Two multiplicative functions $f_{t}$ and $g_{t}$ are said to be equivalent, $f_{t} \sim g_{t}$, if there exists a linear function $\psi_{t}: A \rightarrow A_{0}$ of the form $\psi_{t}(a)=a+\varphi_{1}(a) t+\varphi_{2}(a) t^{2}+\cdots$ such that $f_{t}(a, b)=\psi_{t}^{-1}\left(g_{t}\left(\psi_{t}(a), \psi_{t}(b)\right)\right)$. It is known, see [5], [7], that every deformation given by a multiplicative function $f_{t}$ $=a b+t F_{1}(a, b) \ldots$ is equivalent to a deformation given by a multiplicative function $g_{t}(a, b)=a b+t G_{1}(a, b)+t^{2} G_{2}(a, b) \cdots$ where $G_{i}(a, S)=G_{i}(S, a)=0$ for all $s \in S$ and $a \in A$.

The first theorem we need is due to Kupisch [3].

Received by the editors August 9, 1967. 
THEOREM. Let $A$ be a generalized uniserial basic algebra. Then $A=S+M^{(1)}+M^{(2)}+\cdots+M^{(e)}(K$-direct $)$ where $M^{(1)}+N^{2}=N$ and $M^{(i)}+N^{i+1}=N^{i}$ and $A$ has a basis $\left\{b_{i}\right\}_{i=1}^{n}$ such that $b_{1}=e_{1}, \cdots, b_{p_{0}}$ $=e_{p_{0}}, \quad b_{p_{0}+1} \in N^{(1)} \cdots b_{p_{1}} \in N^{(1)}, \quad b_{p_{1}+1} \cdots b_{p_{2}} \in N^{(2)} \cdots b_{p_{0-1}} \cdots$ $b_{n} \in N^{(e)}$ and the nonzero products of the $b_{p_{i}+1} \cdots b_{p_{i}}$, with the $b_{p_{0+1}}$ $\cdots b_{p_{1}}$ are the $b_{p_{i+1}+1} \cdots b_{p_{i}+2}$ and for each $b_{\alpha}$ there exists a unique $i$ such that $e_{i} b_{\alpha}=b_{\alpha}$ and a unique $j$ such that $b_{\alpha} e_{j}=b_{\alpha}$. We will write $e_{\boldsymbol{i}} m e_{j}$ for the $b_{p_{0+1}} \cdots b_{p_{\mathbf{2}}}$.

We will need also the following lemma the proof of which is routine and will be omitted.

Lemma. Let $K$ be any field and let $A=K[x] / I, I$ any ideal of $K[x]$. Then $A$ is generalized uniserial.

We now prove two lemmas the first of which is a special case of the desired result and the second a reduction theorem.

Lemma. The only deformations of $L[x] /\left(x^{n}\right)$ are of the form $L[x] / P(x)$ where $P(x)=a_{0}+a_{1} x+\cdots+a_{n-1} x^{n-1}+x^{n}$ and $L=K((t))$.

Proof. Since $F_{1} \in H^{2}(A, A)$ we claim $F_{1} \sim G_{1}$ where $G_{1}\left(x, x^{j}\right)=0$ for $j<n-1$. This follows by setting

$$
\phi(1)=0, \quad \phi\left(x^{j}\right)=F_{1}\left(x, x^{j}\right)+x \phi\left(x^{j-1}\right)
$$

and letting $G_{1}=F_{1}-\delta \phi$.

Notice now if we set $g_{t}(a, b)=a b+t G_{1}(a, b)$ we have

$$
A_{\boldsymbol{\sigma}} \cong L[x] /\left(x^{n}+t G_{1}\left(x, x^{n-1}\right)\right) \text {. }
$$

Thus $G_{1}\left(a, G_{1}(b, c)\right)-G_{1}\left(G_{1}(a, b), c\right)=0, a, b, c \in K[x] /\left(x^{n}\right)$.

Now let $f_{t}$ be a multiplicative function of the form

$$
f_{t}(a, b)=a b+t F_{1}(a, b)+t^{2} F_{2}(a, b) \cdots
$$

then by the above $f_{t} \sim f_{t}^{\prime}$ where

$$
f_{\mathfrak{l}}^{\prime}(a, b)=a b+t G_{1}(a, b)+t^{2} G_{2}(a, b) \cdots
$$

and $G_{1}\left(x, x^{j}\right)=0, j<n-1$, and $G(a, G(b, c))-G(G(a, b), c)=0$ for all $a, b, c$, in $K[x] /\left(x^{n}\right)$. By this last equation we see that $\delta G_{2}=0$, so we can repeat the process to $G_{2}$, so that $f_{t} \sim f_{t}^{\prime \prime}$ where

$$
f_{t}^{\prime \prime}(a, b)=a b+t G_{1}(a, b)+t^{2} G_{2}^{\prime}(a, b)+\cdots
$$

where $G_{2}^{\prime}\left(x, x^{j}\right)=0, j<n-1$; and it follows that $G_{3}^{\prime}=0$. Continuing by induction we find $f_{t} \sim h_{t}$ where

$$
h_{t}(a, b)=a b+t H_{1}(a, b)+t^{2} H_{2}(a, b) \cdots
$$


and $H_{i}\left(x, x^{j}\right)=0, j<n-1$. Now set

$$
p(x)=x^{n}+t H_{1}\left(x, x^{n-1}\right)+t^{2} H_{2}\left(x, x^{n-1}\right) \cdots .
$$

One can easily verify that $A_{h_{t}} \cong L[x] /(p(x))$.

Leмma. Let $A$ be a generalized uniserial basic algebra where $N$ $=M+M^{2}+M^{3}+\cdots+M^{n}$ as in Theorem 3.9. Then if $F \in H^{2}(A, A)$ and $F\left(M^{i}, M^{j}\right) \in \sum_{l \geq i+j+1} M^{l}, F$ is trivial.

Proof. Let $e_{i} m e_{j}={ }_{i} m_{j}$ be the basis of $M$ from Kupisch's Theorem. On the basis of $M^{2}$ define

$$
\begin{aligned}
\phi_{1}(m) & =-F\left({ }_{i} n_{j},{ }_{i} n_{k}\right) \quad \text { for } m={ }_{i} m_{j}, m_{k}, \\
& =0 \quad \text { otherwise }
\end{aligned}
$$

and extend $\phi_{1}$ linearly to all of $A$ in the obvious way. Now $G_{1}$ $=F+\delta \phi_{1}$ has the property that $G_{1}(M, M)=0$. Define $\phi_{2}$ on the basis of $M^{3}$ by

$$
\begin{aligned}
\phi_{2}(m) & =-G\left({ }_{i} m_{j} j_{j} m_{k}, k^{m p}\right), \quad m={ }_{i} n_{j}, m_{k k} m_{p}, \\
& =0 \quad \text { otherwise }
\end{aligned}
$$

and extend to all of $A$.

Setting $G_{2}=G_{1}+\delta \phi_{2}, G_{2}$ has the property that $G_{2}\left(M^{2}, M\right)=0$. But since $\delta G_{2}=0, G_{2}\left(M, M^{2}\right)=0$. Continuing inductively we obtain $F \sim G$ where $G(M, N)=0$. It follows that $G=0$ since $\delta G=0$.

TheOREM. If $A$ is a generalized uniserial basic algebra, then all. deformations of $A_{0}$ are generalized uniserial.

Proof. Let $F_{t}$ be a multiplicative function. We can assume that $f_{t}$ has the form $f_{t}(a, b)=a b+t F_{1}(a, b)+t^{2} F_{2}(a, b) \cdots$ and $F_{1}$ is not zero in $H^{2}(A, A)$. Let $F=F_{1}+F_{2}$ where

$$
F_{1}\left(M^{i}, M^{j}\right) \in \sum_{k \leq i+j} M^{k}, \quad F_{2}\left(M^{i}, M^{j}\right) \in \sum_{k \geq i+j+1} M^{k} .
$$

One checks easily that $F_{1}$ and $F_{2} \in H^{2}(A, A)$, and therefore we can assume $F_{2}=0$ by the above.

Now since $F$ is nontrivial it follows that $F(M, N) \neq 0$; so there exists ${ }_{i} m_{j}$ such that $F\left({ }_{i} m_{j}, M^{l}\right) \neq 0$. Pick ${ }_{i} m_{j}$ so $l$ is minimal with this property. Now for some ${ }_{t} m_{k k} m \cdots{ }_{b} m_{p} \in M$ we have

$$
F\left({ }_{i} m_{j},{ }_{j} m_{k k} m \cdots{ }_{b} m_{p}\right)={ }_{i} n_{j j} n_{p} \neq 0,
$$

where ${ }_{j} y_{p} \in \sum_{1 \leq k \leq-1} M^{k}$. Set 


$$
\begin{aligned}
\phi(z) & ={ }_{j} y_{p} \quad z={ }_{j} m_{k k} m \cdots{ }_{b} m_{p}, \\
& =0 \quad \text { otherwise, }
\end{aligned}
$$

extending to all of $A$. Form $F-\delta \phi=G$. $G$ has the property that $G\left(j m_{k}, k m \cdots{ }_{b} m_{p}\right) \neq 0$ and note that ${ }_{k} m_{q} \cdots{ }_{b} m_{p} \in M^{l-1}$. Repeating the process to $G$ we find $F \sim G_{1}$ where $G_{1}\left({ }_{i} m_{j},{ }_{j} m_{p}\right) \neq 0$. But then

$$
G_{1}\left({ }_{i} m_{j},{ }_{j} m_{p}\right)=\alpha, m_{p} \quad \text { some } \alpha \in K,
$$

but by the uniqueness of the idempotents on the left we have $j=i$, which implies $j=p$, which implies $i=p$. So in the above we really had

$$
G_{1}\left({ }_{p} m_{p},{ }_{p} m_{p}\right)=\alpha_{p} m_{p} .
$$

This implies that $A e_{p}$ is a ring direct summand of $A$ and each $F_{i}\left(A e_{p}, A e_{p}\right)$ $\subset A e_{p}$. Now $A e_{p}=e_{p} A e_{p} \cong K[x] /\left(x^{n}\right)$ some $n$; so it follows that we are only deforming ring direct summands which are of the form $K[x] /\left(x^{n}\right)$, and they remain ring direct summands. The result follows.

We now drop all assumptions on $A$ except that $A$ be finite dimensional over $K$.

Theorem. Let $A$ be a Frobenius algebra over $k$. Then every deformation of $A$ is Frobenius as an algebra over $K((t))$.

Proof. Since $A$ is Frobenius there exists a linear functional $\lambda: A \rightarrow K$ such that there are no ideals (right or left) in $\operatorname{ker} \lambda[1$, p. 414]. $A \otimes k((t))$ is Frobenius and in fact if we define $\tilde{\lambda}(a \otimes p(t))$ $=\lambda(a) p(t)$ kernel $\tilde{\lambda}$ contains no ideals in $A \otimes k((t))$. Now let $f_{t}$ be a deformation of $A$. $\boldsymbol{\lambda}$ is still a linear functional on the deformed algebra and one easily checks that kernel $\tilde{\lambda}$ contains no ideals. Thus $A_{f_{t}}$ is Frobenius.

For further theorems on properties perserved under deformations see [5], [6].

\section{BIBLIOGRAPHY}

1. C. W. Curtis and I. Reiner, Representation theory of finite groups and associative algebras, Interscience, New York, 1962.

2. M. Gerstenhaber, On the deformations of rings and algebras, Ann. of Math. 79 (1964), 59-103.

3. H. Kupisch, Symmetrische Algebren mit endlich vielen unzerlegbaren Darstellungen. I, J. für Math. 219 (1965), Heft 1/2.

4. A. Nijenhuis and R. W. Richardson, Jr., Cohomology and deformations of algebraic structures, Bull. Amer. Math. Soc. 72 (1966), 1-29.

5. S. S. Page, On Hochschild extensions and stability of subalgebras in the deformation theory, Ph.D. Thesis, Univ. of Washington, 1966.

6. - Deformations as homomorphic images, (to appear).

7. S. S. Page and R. W. Richardson, Jr., Stable subalgebras of Lie algebras and associative algebras, Trans. Amer. Math. Soc. 127 (1967), 302-312.

University of British Columbia 